\title{
Uso de la tierra y ambiente local de descomposición en el Chaco Semiárido de Córdoba, Argentina
}

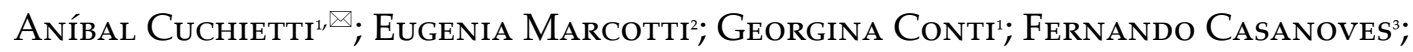 \\ María J. Mazzarino; ${ }^{4}$ María V. Vaieretti ${ }^{1}$; Sandra Díaz ${ }^{1,5}$ \& Natalia Pérez- \\ HARguindegU ${ }^{1,5}$ \\ ${ }^{1}$ Instituto Multidisciplinario de Biología Vegetal (CONICET-UNC). Córdoba, Argentina. ${ }^{2}$ Instituto Argentino de Nivología, \\ Glaciología y Ciencias Ambientales, CONICET, CCT-Mendoza, Mendoza, Argentina. ${ }^{3}$ Centro Agronómico Tropical de \\ Investigación y Enseñanza, CATIE, Costa Rica. ${ }^{4}$ Instituto de Investigaciones en Biodiversidad y Medioambiente (CONICET- \\ CRUB), Bariloche. Río Negro, Argentina. ${ }^{5}$ FCEFyN, Universidad Nacional de Córdoba, Córdoba, Argentina.
}

\begin{abstract}
Resumen. El cambio en el uso de la tierra es un proceso clave debido a sus efectos directos sobre la identidad y la estructura de la vegetación. En el Chaco Semiárido se conoce poco acerca del impacto de los cambios en la vegetación sobre los procesos relacionados con el ciclado de nutrientes (e.g., la descomposición). En este trabajo analizamos si las modificaciones en la vegetación, producto de distintas intensidades en el uso de la tierra, producen cambios en las condiciones del ambiente local, y si estos cambios afectan el patrón de descomposición de dos materiales comunes. En las configuraciones vegetales analizadas encontramos que el cambio en el uso de la tierra tiene un impacto evidente sobre la cobertura vegetal, aunque no se traduce en cambios consistentes en el ambiente local de descomposición. Específicamente, encontramos que el incremento en la intensidad de uso se asoció a la disminución de la cobertura vegetal, de la altura de esa cobertura y de la cantidad de broza depositada sobre el suelo. Estas modificaciones estuvieron vinculadas a una disminución del contenido total de C y N en el suelo. Sin embargo, los cambios en la estructura de la vegetación no se relacionaron con los cambios en las condiciones ambientales locales (temperatura y humedad), pese a que se detectaron diferencias en esas variables ambientales entre las distintas configuraciones vegetales. Por su parte, la descomposición fue mayor en aquellos sitios con menor cobertura vegetal, que a su vez, presentaron menor temperatura del aire. Estos resultados sugieren que el cambio en el uso de la tierra tiene un impacto muy evidente sobre la cobertura de la vegetación, pero este impacto no se traduce en cambios consistentes en el ambiente local de descomposición. En estudios futuros será interesante evaluar la contribución de los procesos de degradación abiótica (fotodegradación y fragmentación física) sobre la descomposición y el ciclado de nutrientes.
\end{abstract}

[Palabras clave: controles de descomposición, materiales comunes, carbono, configuraciones vegetales, estructura de la vegetación, clima local]

\begin{abstract}
Land use and local decomposition environment in the Semiarid Chaco of Córdoba, Argentina. Land use change is a key process due to its direct effects on the identity and structure of vegetation. In semiarid Argentinean Chaco, there is scarse information about the consequences of vegetation changes on processes related to nutrient cycling (e.g., decomposition). In this work, we analyse if changes in local climatic conditions due to changes in vegetation structure, related to different land use types, affect decomposition pattern of two common materials. We found that land use change has a clear impact on vegetation cover, but this impact is not reflected into a consistent change in local decomposition environment. Specifically, we found that more intensive land use types were related to a decrease in vegetation cover and height and also to less litter quantity. These changes were associated with lower soil C and N. However, changes in vegetation structure were not related with changes of local climatic conditions, despite the differences of climatic conditions between vegetal configurations. Decomposition of common materials was faster in vegetal configurations with lower vegetation cover and air temperature. These results suggest that land use change has an evident impact on vegetation cover, but this impact is not transferred on changes of local decomposition environment. In future studies is important to evaluate the contribution of abiotic deterioration processes (photodegradation and physical fragmentation) on decomposition and nutrient cycling.
\end{abstract}

[Keywords: decomposition controls, common materials, carbon, vegetal configurations, vegetation structure, local climate]

Editor asociado: Gervasio Piñeiro

$\triangle$ anibal.cuchietti@gmail.com
Recibido: 9 de septiembre de 2016

Aceptado: 22 de mayo de 2017 


\section{INTRODUCCIÓN}

La comprensión de los factores que regulan la descomposición es esencial para predecir la dinámica de los nutrientes en los ecosistemas (Prescott2010). A escala regional, en numerosos trabajos se ha encontrado que los principales factores que controlan la descomposición son las características climáticas de los ecosistemas, la calidad de la broza y los organismos descomponedores (Coûteaux et al. 1995). A escala local, la estructura de la vegetación y las características edáficas modulan las condiciones del ambiente en el que se produce la descomposición (ambiente local de descomposición), la interfase airesuelo, donde se deposita la broza (Hobbie 1992; Hättenschwiler et al. 2005).

El uso de la tierra puede modificar la identidad y la estructura de la vegetación (i.e., estratificación, cobertura y biomasa), que a su vez pueden afectar tanto el clima local como la cantidad y las características de la broza que llega al suelo y, a través de lo anterior, el contenido de materia orgánica y nutrientes del suelo (Vaieretti et al. 2010; Seidelmann et al. 2016). Por ejemplo, en bosques templados y boreales de Estados Unidos y Canadá, la deforestación genera un aumento en el albedo, que - pese a la disminución de la rugosidad de la superficie vegetal- se asocia a una disminución en la temperatura del aire en los sitios deforestados. Por su parte, en bosques tropicales y subtropicales, el balance entre estos dos componentes es menos claro; incluso, podría generar un aumento en la temperatura del aire en los sitios deforestados (Lee et al. 2011; Anderson-Teixeira et al. 2012). Estos cambios de temperatura del aire pueden influir de manera directa en la temperatura de la interfaz broza-suelo donde se produce la descomposición, y también de manera indirecta al afectar la humedad de la broza y del suelo (Arx et al. 2013). En bosques templados fragmentados del Reino Unido se observó una disminución de la descomposición de leño en áreas más abiertas, cercanas a los bordes de los fragmentos, por el efecto de una mayor temperatura y menor humedad (Crockatt and Bebber 2014). Por su parte, en bosques secos de Costa Rica se encontró que los bosques más viejos con mayor biomasa, menos luz y más humedad (de suelo y broza) favorecen la descomposición (Schilling et al. 2015).

A lo largo de su historia, en el Chaco Semiárido argentino prevalecieron usos de la tierra de bajo impacto (e.g., extracción de madera y ganadería de subsistencia). En las últimas décadas existe un avance acelerado de la agricultura y de la ganadería industrial, pese a las restricciones edáficas y climáticas existentes en ese ecosistema (Zak et al. 2008; Hoyos et al. 2013). Estudios previos realizados en la región muestran que las modificaciones en la vegetación debidas a las diferentes prácticas de manejo afectan a diversos factores controladores del proceso de descomposición. En este sentido, Houspanossian y colaboradores (2013) encontraron que, en sistemas de cultivos del Chaco Semiárido, la temperatura diurna del aire es más alta que en los bosques cercanos. Por su parte, Conti y colaboradores (2014) encontraron que la cantidad de broza acumulada sobre el suelo es menor a medida que aumenta la intensidad de uso. Por otro lado, Abril y Butcher (1999) encontraron que los cambios en la vegetación (bosque, desmonte selectivo, pastizal y matorral) modifican la relación C:N de la broza que producen las distintas configuraciones vegetales. Sin embargo, en ninguno de los estudios antes mencionados se evaluó el efecto de los cambios de los factores controladores sobre el proceso de descomposición en sí mismo.

En este trabajo nos propusimos analizar específicamente si los cambios en las condiciones del ambiente local, resultantes de las modificaciones en la vegetación como producto de distintas intensidades en el uso de la tierra (tala y ganadería actual e histórica), afectan el patrón de descomposición de dos materiales comunes, como indicadores de las condiciones del ambiente local y del potencial de descomposición en diferentes configuraciones vegetales. Para ello, en primer lugar, evaluamos si las configuraciones vegetales asociadas a distintas intensidades de uso presentan diferencias en la estructura de la vegetación, en las condiciones climáticas locales, en las variables edáficas y en el patrón de descomposición de materiales comunes. En segundo lugar, evaluamos si el patrón de descomposición de los materiales comunes se relaciona con cambios en la estructura de la vegetación, en las condiciones climáticas locales o en las variables edáficas.

\section{Materiales y Métodos}

\section{Área de estudio}

El presente estudio se realizó en el extremo suroeste del Chaco Semiárido, al oeste de la provincia de Córdoba, Argentina. El clima de 
la región es subtropical, con una temperatura media anual de $18{ }^{\circ} \mathrm{C}$ y $600 \mathrm{~mm}$ de precipitación media anual (Gorgas and Tassile 2003). En los dos años en los que se realizaron los experimentos se observaron valores de temperatura media anual y precipitación acumulada muy similares a los promedios históricos del área de estudio. Los suelos de la región son aridisoles areno-limosos de origen aluvial del grupo Camborthids (Gorgas and Tassile 2003), con granulometría cada vez más fina en las zonas más bajas (Cora and Bachmeier 2006). La vegetación predominante son bosques xerófilos con un estrato arbóreo dominado por Aspidosperma quebracho blanco y Prosopis flexuosa, y un estrato arbustivo dominado por Mimozyganthus carinatus, Acacia gilliesii y Larrea divaricata (Cabido et al. 1992; Zuloaga et al. 2008).

\section{Selección de configuraciones vegetales, diseño experimental y estructura de la vegetación}

Se seleccionaron cinco configuraciones vegetales muy extendidas en el extremo sur del Chaco Semiárido. Estas configuraciones son el resultado de distintas intensidades en el uso presente y pasado de la tierra (e.g., extracción maderera y pastoreo vacuno y caprino intensivo) sobre la típica configuración de bosque chaqueño de la zona (Cabido and Pacha 2002; Hoyos et al. 2013). Las configuraciones seleccionadas fueron: bosque conservado estratificado (con más de 70 años sin extracción de madera ni ganadería), bosque abierto (tala y ganadería actual no intensivas), arbustal con emergentes (tala selectiva y ganadería vacuna intensiva), arbustal mixto cerrado (tala histórica, ganadería vacuna y caprina intermedia actual) y arbustal abierto dominado por Larrea divaricata (tala histórica, ganadería vacuna y caprina actual de mayor intensidad) (Cuchietti 2016). Las parcelas de cada configuración se seleccionaron en los alrededores de la localidad de Chancaní (c. $31^{\circ} 17^{\prime}-31^{\circ} 50^{\prime} \mathrm{S}$ y $65^{\circ} 16^{\prime}-65^{\circ} 32^{\prime} \mathrm{O}$ ) para mantener una homogeneidad climática regional y las características edáficas generales. En las parcelas seleccionadas no se registraron evidencias presentes o pasadas ni de incendios ni de aplicación de fertilizantes. En cada una de las cinco configuraciones se seleccionaron cuatro parcelas (repeticiones), lo cual hace un total de 20 sitios de muestreo. El tamaño de cada parcela fue de $2500 \mathrm{~m}^{2}$ (50x50 m); dentro de esta superficie se realizaron las mediciones de estructura de la vegetación, de condiciones climáticas locales, de variables edáficas y los experimentos de descomposición. En cada parcela se registró la cobertura vegetal total (\% de cobertura a intervalos de $5 \%$ ) y la altura de la vegetación (m), de acuerdo con la metodología propuesta por Cabido et al. (1992). Además, se determinó la cantidad de broza acumulada en cada configuración vegetal. Para ello, en cada parcela se demarcaron cinco cuadrados de 50x50 cm, dentro de los cuales se recolectó toda la broza que estaba depositada sobre el suelo. Las muestras de broza se secaron en una estufa de circulación forzada a $105^{\circ} \mathrm{C}$ durante 24 horas. Luego, se pesó su contenido y se calculó el promedio de broza acumulada (kg/ $\mathrm{m}^{2}$ ) en cada tipo de configuración vegetal.

\section{Ambiente local de descomposición: condiciones climáticas locales y variables edáficas}

Entre julio de 2009 y mayo de 2011 se registraron las condiciones climáticas locales. Por un lado, en una parcela de cada configuración se midió temperatura y humedad ambiental con sensores HOBO dataloggers (Onset Computer Corporation). Los sensores se ubicaron dentro de recipientes plásticos con aberturas en la parte inferior, sujetos a la rama de un árbol o arbusto a $\sim 1.5 \mathrm{~m}$ de altura. Por otro lado, en todas las parcelas se midió la temperatura del suelo en los primeros $5 \mathrm{~cm}$ de profundidad con sensores $\mathrm{HOBO}$ también colocados dentro de recipientes plásticos, pero cerrados de forma hermética para evitar daños. Todos los sensores se colocaron a no más de $1 \mathrm{~m}$ del sector donde se instalaron las camas de descomposición de los materiales comunes, y se programaron para registrar (cada ocho horas) la temperatura y la humedad ambiental, y la temperatura del suelo. La humedad del suelo se determinó a través del método gravimétrico (Rundel and Jarrell 1989). Para ello, en cada parcela, cada tres meses se recolectó una muestra compuesta (5 subrepeticiones) de los primeros $10 \mathrm{~cm}$ de suelo, que se colocó en un recipiente plástico hermético para evitar pérdida de humedad. Una vez en el laboratorio, las muestras fueron tamizadas (malla de $2 \mathrm{~mm}$ ), pesadas y colocadas en una estufa de circulación forzada durante 48 horas a $105^{\circ} \mathrm{C}$. Luego, las muestras secas fueron pesadas nuevamente para obtener el peso final y determinar el porcentaje de humedad.

Para las mediciones de las variables edáficas se tomaron muestras compuestas de 3 subrepeticiones a $0-10 \mathrm{~cm}$ de profundidad del suelo utilizando un cilindro de $10 \mathrm{~cm}$ 
de diámetro en todas las parcelas. Las subrepeticiones se recolectaron en puntos cercanos a la cama de descomposición de los materiales comunes para captar mejor las características del suelo cercano a la cama de descomposición. Las muestras de suelo fueron secadas en una estufa de circulación forzada y tamizadas (malla de $2 \mathrm{~mm}$ ) para su posterior análisis físico-químico. Las técnicas utilizadas fueron: Day (1986) para textura (\%arena, \%limo y \%arcilla), Thomas (1996) para $\mathrm{pH}$, Walkley-Black para el porcentaje de carbono (\%C) (Nelson and Sommers 1996), Kjeldahl para el porcentaje de nitrógeno total (\%N total) (Bremner 1996), y Bray and Kurtz № 1 para $\mathrm{P}$ (mg/kg) (Kuo 1996). Además, se determinó la compactación del suelo de cada parcela mediante la inserción de un penetrómetro de bolsillo (Forestry Suppliers Inc.) en 20 puntos elegidos al azar hasta una profundidad de 0.7 $\mathrm{cm}$. La compactación se expresó en kilogramos de fuerza por centímetro cuadrado de suelo $\left(\mathrm{kg}_{\mathrm{f}} / \mathrm{cm}^{2}\right)$.

\section{Descomposición de materiales comunes}

Para evaluar el efecto de las condiciones ambientales locales sobre el patrón de descomposición en cada configuración vegetal se utilizaron materiales comunes. El uso de estos materiales permite analizar el efecto integrado de múltiples variables del ambiente sobre la tasa de descomposición, independientemente de la calidad del sustrato a descomponer, ya que el sustrato es común a todos los sitios (O'lear et al. 1996; Orwin et al. 2006). Como materiales comunes se seleccionó la broza de dos especies de la región chaqueña, de calidad contrastante, que no están presentes en el área de estudio (para evitar efectos de afinidad entre la broza y el ambiente local) (Vivanco and Austin 2008). De ambas especies se recolectó broza en la misma estación de crecimiento, compuesta sólo por hojas senescentes levemente unidas a la planta o recientemente caídas sobre el suelo, que no presentaban una coloración verdosa o síntomas evidentes de descomposición. Las especies seleccionadas fueron: Acalypha communis Mull. Arg. (especie de descomposición relativamente rápida) y Jarava ichu Ruiz and Pav. var. ichu (especie de descomposición relativamente lenta) (Vaieretti et al. 2005; Cuchietti et al. 2014).

La descomposición de los materiales comunes se determinó usando la técnica de bolsas de broza, que permite evaluar la disminución de peso a través del tiempo con una pérdida mínima de material (Cornelissen 1996; Pérez-Harguindeguy et al. 2013). Se prepararon 400 bolsas (200 por especie) con una malla de nylon de $0.3 \mathrm{~mm}$. En las bolsas se colocó $1.0 \pm 0.01 \mathrm{~g}$ de material. Aunque las bolsas de malla pequeña sólo permiten el paso de parte de la mesofauna, se considera que la contribución de estos organismos a la tasa de descomposición es relativamente pequeña comparada con la actividad de bacterias, protozoos, hongos y microfauna (Pérez-Harguindeguy et al. 1997; Vaieretti et al. 2010). La broza de ambas especies se conservó en el laboratorio en bolsas de papel madera a temperatura constante para evitar que se deteriorara hasta ser utilizada para cada uno de los experimentos.

Las bolsas se incubaron en camas de descomposición construidas en cada parcela. Para ello, bajo el dosel de las especies leñosas perennes dominantes en cada configuración vegetal se seleccionó un área de $1 \mathrm{~m}^{2}(0.5 \times 2 \mathrm{~m})$, que fue limpiada de restos de plantas, troncos y broza preexistentes. Las bolsas se colocaron sobre la superficie del suelo y para simular las condiciones naturales de descomposición de cada una de las parcelas fueron cubiertas levemente con las ramas y broza previamente removidas. La incubación de las bolsas se realizó durante dos años consecutivos (mayo 2009 a abril de 2010 y mayo 2010 a abril de 2011), bajo las condiciones naturales de temperatura y humedad de cada parcela. Se pusieron a incubar 200 bolsas (100 bolsas por especie) en cada año y se recolectaron a los 90, 180, 270 y 360 días desde el inicio de la incubación. En cada fecha de cosecha se removieron al azar 5 bolsas de cada material, las cuales se mantuvieron a $-14{ }^{\circ} \mathrm{C}$ hasta su procesamiento. Una vez que las muestras fueron descongeladas, se las limpió a mano para remover el suelo adherido y cualquier material extraño. Luego, las muestras fueron secadas en estufa a $60^{\circ} \mathrm{C}$ durante 48 horas. La tasa de descomposición se definió, como en la mayoría de los trabajos sobre la temática, como el porcentaje de $\mathrm{C}$ remanente $\left(\% \mathrm{C}_{\mathrm{Rem}}\right)$ luego de cada fecha de incubación. Se determinó el porcentaje de cenizas para todas las muestras como factor de corrección por contaminación de las muestras con suelo durante la incubación (Harmon et al. 2000; Vivanco and Austin 2006; Furey et al. 2014). Además, para estimar el contenido inicial de agua de la broza se seleccionaron submuestras de los materiales comunes; estas submuestras fueron secadas en estufa de circulación forzada a $60{ }^{\circ} \mathrm{C}$ durante 48 horas. Luego, se incorporó la diferencia 
de peso como un factor de corrección del peso inicial de cada muestra utilizada en los experimentos de descomposición.

\section{Análisis de datos}

Las variables de estructura de la vegetación y las variables edáficas se analizaron mediante ANAVA. Cuando las diferencias fueron significativas se realizó la prueba a posteriori de Tukey (Sokal and Rohlf 1995). La temperatura del aire, la humedad ambiental y la temperatura del suelo en función del tiempo se analizaron utilizando diagramas de dispersión, identificando las distintas configuraciones vegetales. Para determinar las diferencias entre las configuraciones se realizaron contrastes. Los sensores utilizados para registrar la temperatura del suelo mostraron discontinuidades en el registro de los datos, por lo que no fue posible tener el número necesario de repeticiones para determinar la varianza en esta variable. Para analizar las diferencias en la humedad del suelo y en la tasa de descomposición $\left(\% \mathrm{C}_{\mathrm{rem}}\right)$ de los materiales comunes entre cada una de las configuraciones vegetales se realizaron modelos lineales generales y mixtos para considerar la heterogeneidad de varianza durante el período analizado. Como los experimentos fueron repetidos en el tiempo (4 fechas de cosecha), se consideraron distintos tipos de estructura de las matrices de varianza y covarianza (Di Rienzo et al. 2011). Este análisis se realizó para los dos años de muestreo separadamente. Luego se seleccionó el modelo más apropiado utilizando los valores del criterio de Akaike (AIC), Schwarz (BIC) y la prueba de máxima verosimilitud (LRT).

Se emplearon análisis de correlación para evaluar las asociaciones entre las variables de estructura de la vegetación, condiciones ambientales, edáficas, y los patrones de descomposición. Para el caso de las asociaciones con la temperatura, la humedad del aire y la temperatura del suelo se utilizó un promedio para cada configuración $(n=5)$, para cada año de muestreo por separado en el caso correspondiente, ya que sólo se dispuso de un sensor de temperatura y humedad del aire por configuración. Para el caso de las asociaciones entre las variables de estructura de la vegetación, edáficas, humedad del suelo y descomposición se utilizaron promedios de cada parcela $(n=20)$. Esta última aproximación permite evaluar la asociación de las variables independientemente de la configuración a la que pertenecen las parcelas. Para todas las variables medidas se analizó la normalidad usando la prueba de Shapiro-Wilks (Sokal and Rohlf 1995). Todos los análisis estadísticos se realizaron utilizando el programa InfoStat v. 2015 (Di Rienzo et al. 2015).

\section{Resultados}

\section{Estructura de la vegetación, condiciones climáticas locales y variables edáficas}

La estructura de la vegetación presentó diferencias significativas entre las configuraciones vegetales. La cobertura total fue mayor en el arbustal mixto cerrado y menor en el arbustal con emergentes y el arbustal abierto $(F=11.68 ; P \leq 0.0002)$. La altura de la vegetación fue mayor en el bosque conservado y menor en el arbustal mixto cerrado y el arbustal abierto $(F=21.96$; $P \leq 0.0001)$. Por último, la cantidad de broza depositada sobre el suelo, fue mayor en el bosque conservado que en el arbustal abierto $(F=13.91 ; P \leq 0.0003)$ (Tabla 1$)$.

Las condiciones climáticas locales entre las distintas fisonomías vegetales durante todo el período analizado (dos años, 20092011) presentaron variaciones significativas entre algunas configuraciones (Figura 1). La temperatura media del aire fue más alta en el arbustal mixto cerrado que en el arbustal con emergentes (Figura 1a). La temperatura media del suelo fue alrededor de $3^{\circ} \mathrm{C}$ más alta en el arbustal mixto cerrado que en el bosque conservado y el arbustal con emergentes (Figura 1b). Por otro lado, la humedad relativa ambiental fue mayor en el arbustal con emergentes y menor en el arbustal mixto cerrado (Figura 1c). Por último, la humedad de suelo fue mayor en el arbustal con emergentes y menor en el arbustal mixto cerrado (Figura 1d).

Noseencontraron diferenciassignificativasen la textura (arena, limo y arcilla) y tampoco en el valor de $\mathrm{pH}$ del suelo entre las configuraciones vegetales (Tabla 1). El contenido de C del suelo fue mayor en el bosque conservado y el bosque abierto y menor en el arbustal abierto $(F=10.46$; $P \leq 0.0004)$. El contenido de $\mathrm{N}$ total del suelo mostró una tendencia similar al contenido de $\mathrm{C}$, fue mayor en el bosque conservado y el bosque abierto y menor en el arbustal abierto $(F=4.46 ; P \leq 0.0200)$. El contenido de $\mathrm{P}$ del suelo fue mayor en al arbustal con 
Tabla 1. Estructura de la vegetación, condiciones climáticas locales y variables edáficas. En cada celda se muestran los promedios y los errores estándar de las variables analizadas. En las columnas se indican los valores de las variables para cada configuración vegetal: bosque conservado (BosCo), bosque abierto (BosAb), arbustal con emergentes (ArbEm), arbustal mixto cerrado (ArbMi) y arbustal abierto (ArbAb). En las filas se indican las distintas variables analizadas de la estructura de la vegetación, el clima local y edáficas. Abreviaturas: temperatura (Temp), humedad (Hum), carbono $(\mathrm{C})$, nitrógeno $(\mathrm{N})$ y fósforo $(\mathrm{P})$. Letras distintas indican diferencias significativas entre configuraciones vegetales (para la estructura de vegetación, variables edáficas y humedad del suelo se realizó una prueba de Tukey, $P \leq 0.05$; para el resto de las condiciones climáticas locales se realizaron contrastes entre todas las configuraciones las diferencias significativas se especifican en el texto).

Table 1. Vegetation structure, local climatic conditions and edaphic variables. Each cell shows the analysed variables means and standard errors between brackets. Each column shows the values for each variable in the vegetal physiognomies. The different plant physiognomies analysed were: preserved forest (BosCo), open forest (BosAb), shrubland with emerging trees (ArbEm), mixed shrubland (ArbMi) and open shrubland (ArbAb). Each row shows the analysed variables of vegetal structure, local climatic conditions and edaphic. Abbreviations: temperature (Temp), humidity (Hum), carbon (C), nitrogen $(\mathrm{N})$ and phosphorus $(\mathrm{P})$. Different letters indicate significant differences between vegetal physiognomies (Tukey test; $P \leq 0.05$ for vegetal structure and edaphic variables and soil humidity. Contrasts for air and soil temperature and air humidity, significant differences between plant physiognomies are specify in the text).

\begin{tabular}{lccccc}
\hline & \multicolumn{4}{c}{ Configuración vegetal } \\
& BosCo & BosAb & ArbMi & ArbEm & ArbAb \\
\hline Estructura de la vegetación & & & & \\
$\quad$ Cobertura total $(\%)$ & $70(2) \mathrm{ab}$ & $60(4) \mathrm{bc}$ & $80(4) \mathrm{a}$ & $53(5) \mathrm{c}$ & $48(4) \mathrm{c}$ \\
Altura vegetación $(\mathrm{m})$ & $12.4(0.3) \mathrm{a}$ & $12.3(0.5) \mathrm{ab}$ & $7.6(1) \mathrm{c}$ & $8.8(1) \mathrm{bc}$ & $5.4(0.3) \mathrm{c}$ \\
Cantidad de broza $\left(\mathrm{kg} / \mathrm{m}^{2}\right)$ & $2222(243) \mathrm{a}$ & $1680(111) \mathrm{ab}$ & $1291(62) \mathrm{bc}$ & $1822(340) \mathrm{ab}$ & $693(25) \mathrm{c}$ \\
Condiciones climáticas locales & & & & \\
Temp. del aire $\left({ }^{\circ} \mathrm{C}\right)$ & $23.4(2.7)$ & $23.8(2.5)$ & $24.2(2.6)$ & $22.5(2.2)$ & $23.1(2.5)$ \\
Hum. relativa ambiental $(\%)$ & $46.3(3.1)$ & $46.2(2.9)$ & $43.4(3)$ & $47.6(3.4)$ & $46.3(2.9)$ \\
Temp. del suelo $\left({ }^{\circ} \mathrm{C}\right)$ & $22.5(2.3)$ & $23.4(2.8)$ & $25.7(2.2)$ & $22.5(2.7)$ & $24.4(3.2)$ \\
Hum. del suelo $(\%)$ & $2.7(0.3) \mathrm{b}$ & $2.3(0.2) \mathrm{bc}$ & $2(0.2) \mathrm{c}$ & $5.5(0.3) \mathrm{a}$ & $2.3(0.2) \mathrm{bc}$ \\
Variables edáficas & & & & \\
Arena $(\%)$ & $59.1(1.4) \mathrm{a}$ & $59.1(1.3) \mathrm{a}$ & $54.0(2.9) \mathrm{a}$ & $52.0(1.8) \mathrm{a}$ & $57.8(3.2) \mathrm{a}$ \\
Limo $(\%)$ & $28.6(1.3) \mathrm{a}$ & $28.7(1.2) \mathrm{a}$ & $33.4(2.6) \mathrm{a}$ & $35.7(1.7) \mathrm{a}$ & $32.5(2.8) \mathrm{a}$ \\
Arcilla $(\%)$ & $12.4(0.4) \mathrm{a}$ & $12.2(0.4) \mathrm{a}$ & $12.7(0.6) \mathrm{a}$ & $12.3(1.4) \mathrm{a}$ & $9.8(0.7) \mathrm{a}$ \\
pH & $6.9(0.1) \mathrm{a}$ & $6.7(0.4) \mathrm{a}$ & $7.1(0.2) \mathrm{a}$ & $6.6(0.3) \mathrm{a}$ & $7.0(0.2) \mathrm{a}$ \\
$\mathrm{C}(\%)$ & $1.63(0.08) \mathrm{a}$ & $1.60(0.14) \mathrm{a}$ & $0.97(0.15) \mathrm{bc}$ & $1.41(0.85) \mathrm{ab}$ & $0.67(0.03) \mathrm{c}$ \\
$\mathrm{N}$ total $(\%)$ & $0.17(0.01) \mathrm{a}$ & $0.17(0.04) \mathrm{a}$ & $0.13(0.02) \mathrm{ab}$ & $0.14(0.08) \mathrm{ab}$ & $0.08(0.01) \mathrm{b}$ \\
$\mathrm{P}(\mathrm{mg} / \mathrm{kg})$ & $30.6(7.6) \mathrm{ab}$ & $15.7(6.4) \mathrm{b}$ & $14.8(2.2) \mathrm{b}$ & $42.7(7.2) \mathrm{a}$ & $18(2.6) \mathrm{ab}$ \\
Compactación $\left(\mathrm{kg}_{\mathrm{f}} / \mathrm{cm}^{2}\right)$ & $0.8(0.4) \mathrm{b}$ & $1.2(0.3) \mathrm{ab}$ & $1.6(0.3) \mathrm{ab}$ & $1.8(0.4) \mathrm{ab}$ & $2.8(0.4) \mathrm{a}$ \\
\hline
\end{tabular}

emergentes y menor en el bosque abierto y el arbustal mixto cerrado $(F=4.65 ; P \leq 0.0100)$. Por último, la compactación del suelo fue mayor en el arbustal abierto y menor en el bosque conservado ( $F=3.41 ; P \leq 0.0400)$ (Tabla 1$)$.

\section{Relación de la estructura de la vegetación con las condiciones climáticas locales y las variables edáficas}

Ninguna de las variables de estructura de la vegetación, ni tampoco de las variables edáficas, se relacionó con las variables descriptoras de las condiciones climáticas locales. Sí se encontraron asociaciones entre la estructura de la vegetación y ciertas variables edáficas. A medida que la cantidad de broza fue mayor, la cantidad de $\mathrm{C}$ en el suelo fue mayor (Figura 2). Asimismo, a medida que aumentó la altura de la vegetación la cantidad de broza, el contenido de C y el contenido de
$\mathrm{N}$ en el suelo fueron mayores (Figura 2). Finalmente, a mayor cobertura y altura de la vegetación, la compactación del suelo fue menor (Figura 2). El resto de las variables edáficas medidas no se relacionaron con las variables de estructura de la vegetación.

\section{Descomposición de los materiales comunes}

Tal como se esperaba de acuerdo a la calidad inicial de cada uno de los materiales comunes, el \%C $\mathrm{C}_{\text {rem }}$ de A. communis fue menor al de J. ichu (Figura 3) en todas las fisonomías vegetales en las dos temporadas de incubación. En base al periodo completo de incubación (modelos lineales generales y mixtos), en la primera temporada el $\% \mathrm{C}_{\mathrm{rem}}$ de $A$. communis fue significativamente menor en el arbustal abierto, el arbustal con emergentes y el bosque abierto que en el bosque conservado y el arbustal mixto cerrado $(F=16.22 ; P \leq 0.0001)$ 


\section{a)}

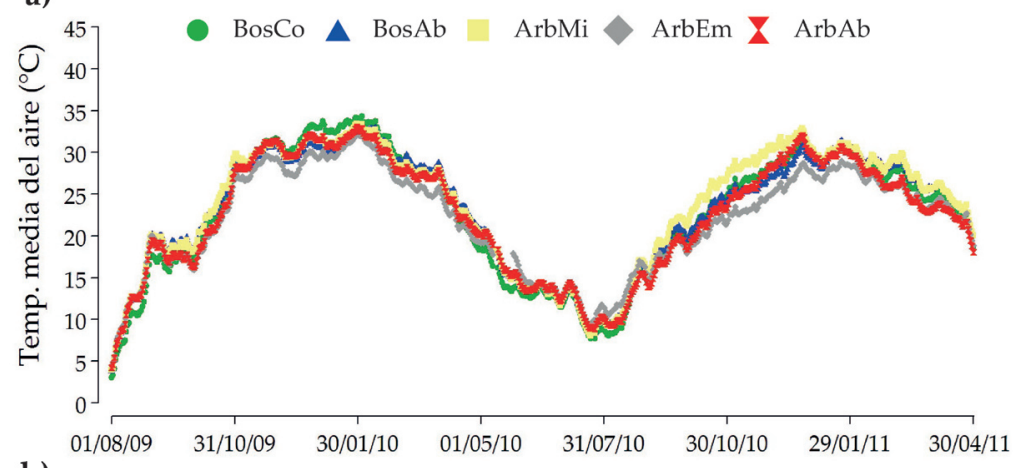

b)
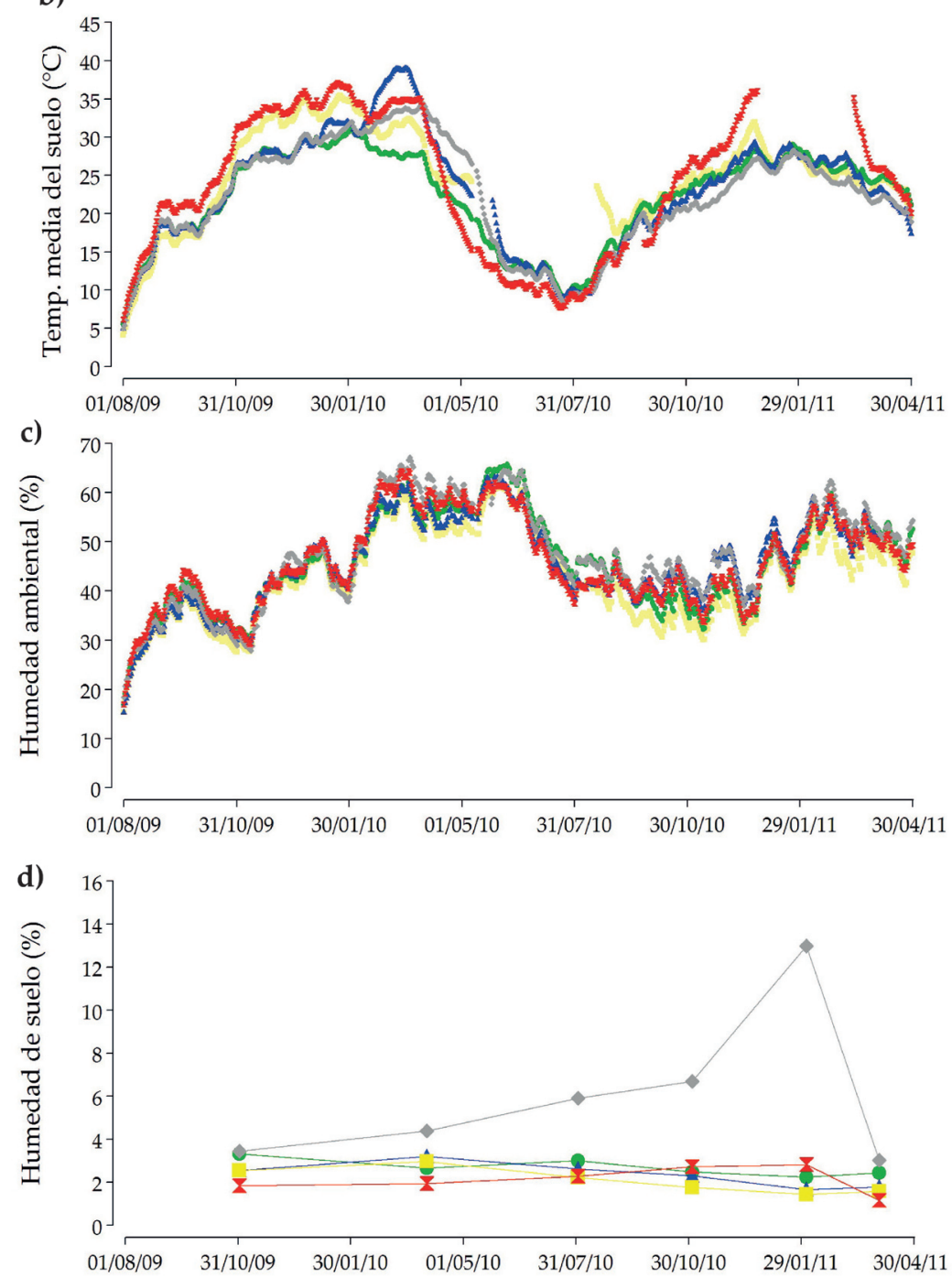

Figura 1. Condiciones climáticas locales durante todo el periodo analizado (2009-2011). Las variables analizadas en las distintas configuraciones vegetales fueron: a) temperatura media del aire $\left.\left({ }^{\circ} \mathrm{C}\right), \mathrm{b}\right)$ temperatura media del suelo $\left.\left({ }^{\circ} \mathrm{C}\right), \mathrm{c}\right)$ humedad relativa ambiental (\%) y d) humedad de suelo (\%). Cada símbolo corresponde a un valor diario de temperatura y la humedad relativa ambiental determinados con sensores $\mathrm{HOBO}$, mientras que los valores de humedad de suelo se determinaron por método gravimétrico.

Figure 1. Local climatic conditions during the analyze period (2009-2011). The variables measured at different plant physiognomies were: a) mean air temperature $\left({ }^{\circ} \mathrm{C}\right)$, b) mean soil temperature $\left.\left({ }^{\circ} \mathrm{C}\right), \mathrm{c}\right)$ ambient relative humidity $(\%)$ and d) soil humidity (\%). Each symbol corresponds to a daily value of temperature and ambient relative humidity measured with $\mathrm{HOBO}$ data loggers, while soil humidity values were measured using the gravimetric method. 


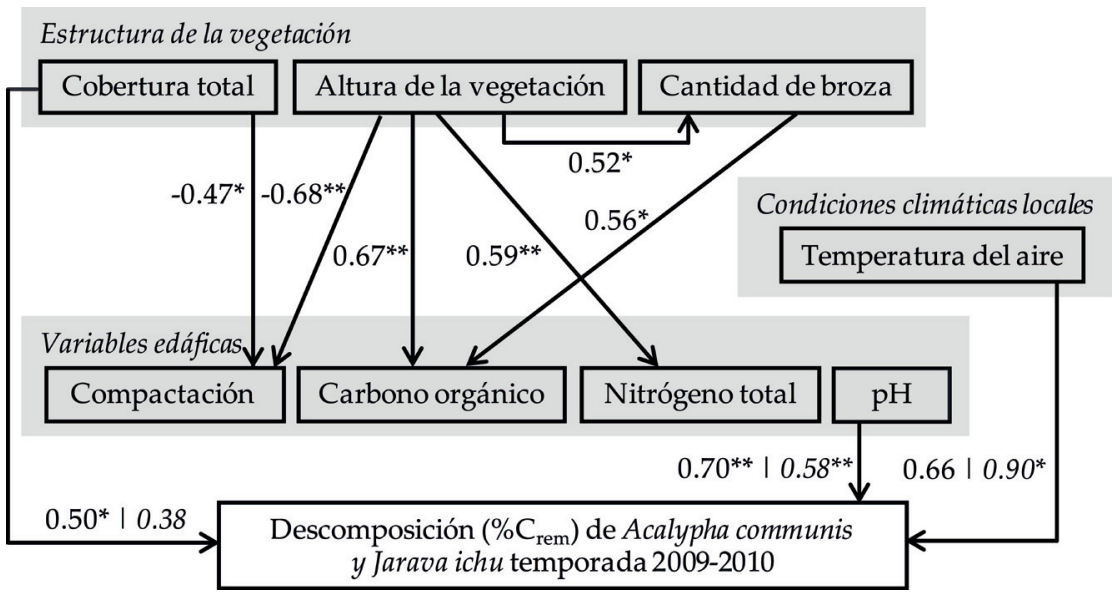

Figura 2. Diagrama de correlaciones entre la estructura de la vegetación, las condiciones climáticas locales, las variables edáficas y la descomposición de los materiales comunes $\left(\% \mathrm{C}_{\mathrm{rem}}\right)$. Los valores separados por una línea vertical indican el coeficiente de correlación de Pearson, a la izquierda para Acalypha communis y a la derecha para Jarava ichu. Las correlaciones entre la estructura de la vegetación, las variables edáficas y la descomposición tienen un $n=20$, mientras que entre las variables climáticas y la descomposición tienen un $n=5$. Los asteriscos indican los valores de significancia $\left({ }^{*} P \leq 0.05 ; * * P \leq 0.01\right)$.

Figure 2. Diagram of correlations between vegetation structure, local environmental conditions, soil variables and common materials decomposition $\left(\% \mathrm{C}_{\mathrm{rem}}\right)$. The values separated by a vertical line indicate Pearson correlation coefficient, in the left for Acalypha communis and in the right for Jarava ichu. Correlations between vegetation structure, soil variables and decomposition have an $n=20$, while the correlation between local environment conditions and decomposition have an $n=5$. Asterisks indicate significance values $\left({ }^{*} P \leq 0.05\right.$; $\left.{ }^{* *} P \leq 0.01\right)$.
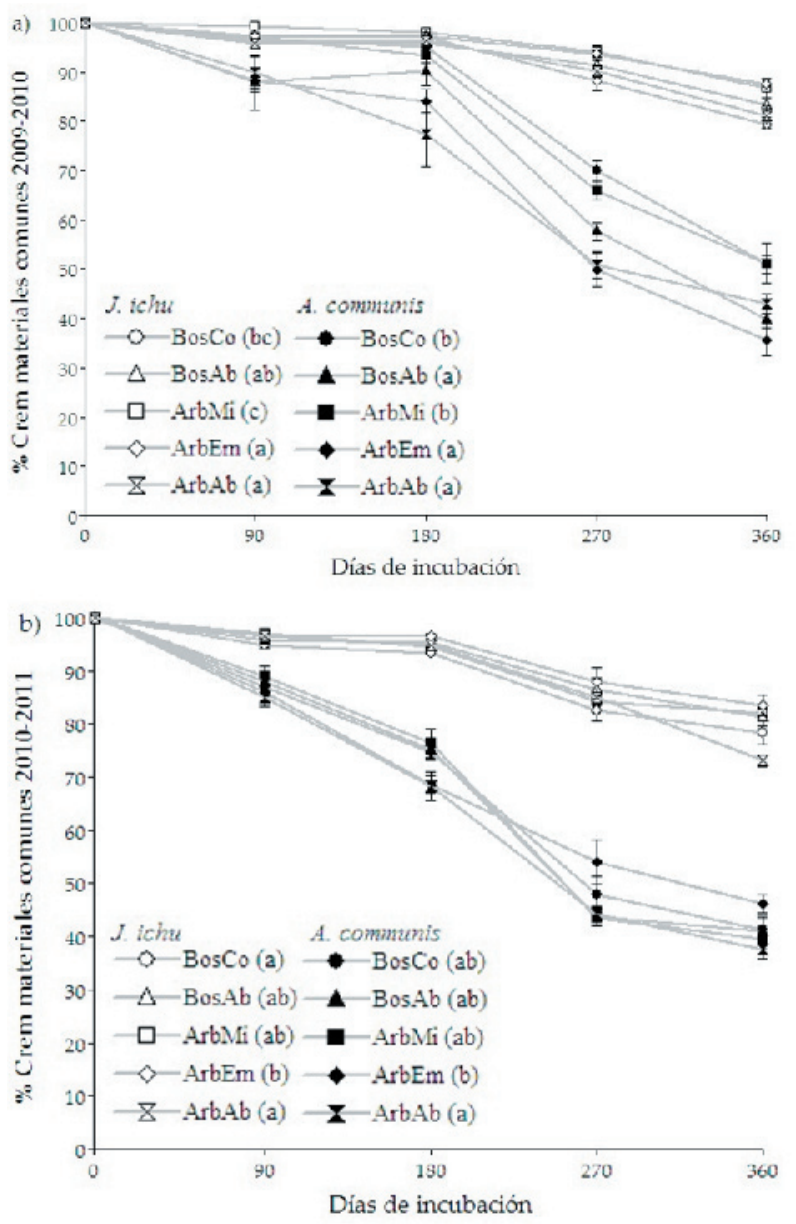

Figura 3. Patrón de descomposición de los materiales comunes $\left(\% \mathrm{C}_{\mathrm{rem}}\right)$ en las distintas configuraciones vegetales: a) de mayo de 2009 a abril de 2010 y b) de mayo de 2010 a abril de 2011. Los símbolos blancos indican la descomposición de Jarava ichu y los negros la de Acalypha communis. Los distintos símbolos indican las distintas configuraciones vegetales: bosque conservado (círculo), bosque abierto (triángulo), arbustal mixto cerrado (cuadrado), arbustal con emergentes (rombo) y arbustal abierto (reloj de arena).

Figure 3. Decomposition pattern of common materials $\left(\% \mathrm{C}_{\text {rem }}\right)$ at different plant physiognomies: a) From May 2009 to April 2010 and b) from May 2010 to April 2011. The white symbols indicate decomposition of Jarava ichu and black symbols decomposition of Acalypha communis. The different symbols indicate the different plant physiognomies: preserved forest (circle), open forest (triangle) closed mixed shrubland (square), shrubland with emerging trees (diamond) and open shrubland (sandclock). 

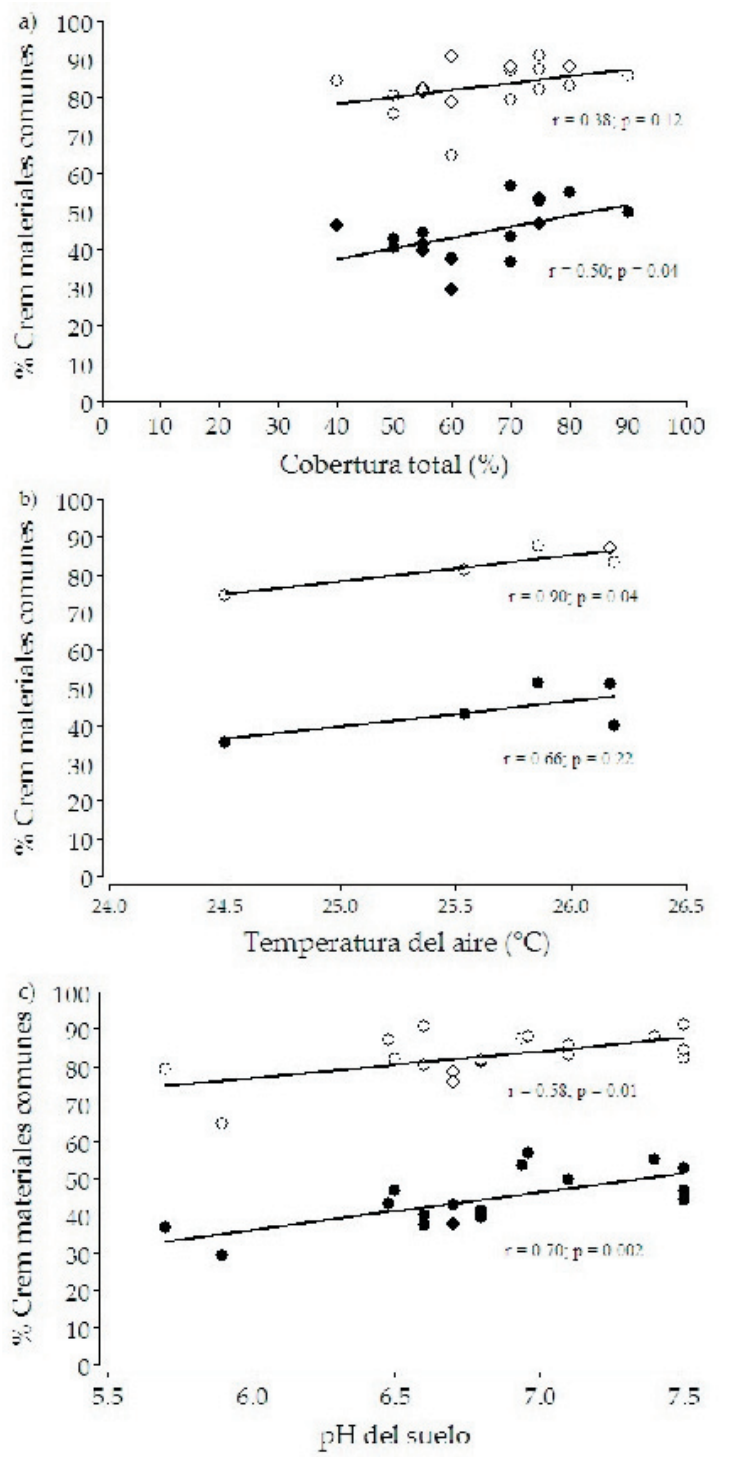

Figura 4. Asociaciones entre el porcentaje de carbono remante $\left(\% \mathrm{C}_{\text {rem }}\right)$ de ambos materiales en el período 2009-2010 y a) porcentaje de cobertura total, b) media de temperatura del aire en el mismo período y c) $\mathrm{pH}$ del suelo. Los círculos negros corresponden a Acalypha communis y los círculos blancos a Jarava ichu.

Figure 4. Associations between the percentage of remnant carbon $\left(\% \mathrm{C}_{\mathrm{rem}}\right)$ of both standard materials in the incubation period of 2009-2010 and: a) total vegetation cover $(\%), b)$ mean air temperature in the same period and c) soil pH. Black circles correspond to Acalypha communis and white circles to Jarava ichu.

(Figura 3a). Mientras que durante el segundo período de incubación, $\mathrm{el} \% \mathrm{C}_{\mathrm{rem}}$ de $A$. communis fue significativamente menor en el arbustal abierto que en el arbustal con emergentes $(F=3 ; P \leq 0.0300)$ (Figura $3 b)$. Por otro lado, $J$. $i c h u$ presentó diferencias entre las fisonomías vegetales similares a $A$. communis, durante el primer período de incubación el \% $\mathrm{C}_{\text {r }}$ fue significativamente menor en el arbustal con emergentes y el arbustal abierto que en el arbustal mixto y el bosque conservado $(F=16.22 ; P \leq 0.0001)$ (Figura 3a). Mientras tanto, durante el segundo período de incubación, el $\% \mathrm{C}_{\text {rem }}$ de $J$. ichu fue significativamente menor en el bosque conservado y el arbustal abierto que en el arbustal con emergentes $(F=3$; $P \leq 0.0300$ ) (Figura 3b).

Descomposición de los materiales comunes y su relación con los controles de la descomposición

En base a los valores finales del proceso de descomposición se encontró que durante el primer período de incubación (2009$2010)$, el $\% \mathrm{C}_{\mathrm{rem}}$ de $A$. communis fue menor en parcelas con menor cobertura total de la vegetación $(\mathrm{r}=0.50 ; P \leq 0.0400 ; \mathrm{n}=20)$ (Figura $4 \mathrm{a})$, mientras que el $\% \mathrm{C}_{\text {rem }}$ de J. ichu no se asoció con la cobertura total $(\mathrm{r}=0.38 ; P \leq 0.1200$; $\mathrm{n}=20$ ) (Figura $4 \mathrm{a}$ ). Por su parte, el $\% \mathrm{C}_{\text {rem }}$ de $J$. ichu fue menor en aquellas parcelas que presentaron menor temperatura del aire $(\mathrm{r}=0.90 ; P \leq 0.0400 ; \mathrm{n}=5)$ (Figura $4 \mathrm{~b})$, mientras que el $\% \mathrm{C}_{\text {rem }}$ de $A$. communis no se asoció con esta variable $(\mathrm{r}=0.66 ; P \leq 0.2200 ; \mathrm{n}=20)$ (Figura $4 b)$. Por último, los dos materiales comunes presentaron un menor $\% \mathrm{C}_{\text {rem }}$ en parcelas con menor $\mathrm{pH}$ del suelo $(\mathrm{r}=0.70 ; P \leq 0.0020$ para $A$. communis; $\mathrm{r}=0.58 ; P \leq 0.0100$ para J. ichu; $\mathrm{n}=20$ ) (Figura 4c). Mientras tanto, durante el segundo período de incubación (2010-2011), el \% de los dos materiales comunes no mostró asociaciones con ninguna de las variables de estructura de la vegetación, condiciones del ambiente local, o edáficas.

\section{Discusión}

En el presente trabajo encontramos que efectivamente distinta intensidad en el uso de la tierra (i.e., tala y ganadería históricas y actuales) se asoció a una disminución de la cobertura vegetal, de la altura de la misma y de la cantidad de broza depositada sobre el suelo. A su vez, estas modificaciones en la vegetación estuvieron asociadas a una disminución del contenido total de $\mathrm{C}$ y $\mathrm{N}$ en el suelo. Los cambios en la estructura de la vegetación no estuvieron asociados a los cambios en las condiciones ambientales locales, pese a que se detectaron diferencias en esas condiciones entre las distintas configuraciones vegetales. Por su parte, el patrón de descomposición de los materiales comunes también presentó diferencias entre las configuraciones vegetales asociadas al uso de la tierra y se relacionó tanto con la cobertura total de la vegetación como 
con la temperatura del aire. En este sentido, la descomposición fue mayor en aquellos sitios con menor cobertura vegetal, los que a su vez presentaron menor temperatura del aire y que, en general, corresponden a los sitios con uso más intensivo. Estas asociaciones entre el patrón de descomposición y las variables del ambiente local no fueron consistentes en los dos períodos evaluados.

Tal como se ha mostrado para otros sistemas (Paudel et al.2015), como así también para la región de estudio (Zak et al. 2008; Karlin et al. 2013), las prácticas de manejo tanto actuales como históricas relacionadas a la tala y a la cría de ganado doméstico generan modificaciones fuertes en la estructura y cobertura de la vegetación. Existen evidencias de que los cambios en la cobertura de la vegetación pueden modificar las condiciones climáticas a escala local, regional y global (Adams 2007; Lee et al. 2011; Anderson-Teixeira et al. 2012; Houspanossian et al. 2013; Flerchinger et al. 2015). A su vez, pueden tener efectos sobre la interfaz broza-suelo donde se produce la descomposición (Eviner and Chapin 2003; Yates et al. 2000; Schiling et al. 2016 o similares). En las configuraciones vegetales analizadas durante los años estudiados (2009-2011), la mayor temperatura del aire observada en el arbustal mixto cerrado y los bosques puede deberse a que el menor albedo que poseerían (Lee et al. 2011; AndersonTeixeira 2012) no se vea compensado por su mayor rugosidad (y su mayor disipación de calor latente), como podría esperarse en base a estudios previos (Houspanossian et al. 2013). A su vez, una mayor temperatura podría deberse a que en arbustales y bosques existe una mayor superficie oscura de troncos, ramas y broza sobre el suelo, que podría retener calor incluso durante la noche, cuando el efecto de la rugosidad en disipar calor sería más importante (Eviner and Chapin 2003; Adams 2007). En particular, la retención de calor podría ser significativa bajo el dosel; así afectaría a la interfaz broza-suelo donde ocurre la descomposición debido a que el aire bajo el dosel es menos mezclado por el viento que el aire sobre las copas (Adams 2007; Flerchinger et al. 2015).

En relación a la humedad del suelo, es probable que la amplia variación espacio temporal característica de los ambientes semiáridos (Longobardi 2008; Jin et al. 2011; Deng et al. 2016) no haya podido ser detectada por las mediciones trimensuales realizadas. De existir una asociación entre la humedad del suelo y la descomposición, debe ser evaluada en el contexto del efecto de los pulsos de lluvia (Austin et al. 2004). La caída de precipitaciones en el sistema de estudio es relativamente escasa y concentrada en los meses estivales (Gorgas and Tassile 2003); además, las altas temperaturas diurnas en las configuraciones analizadas producen una rápida evaporación del agua caída (Karlin et al. 2013). La humedad relativa del suelo de cada configuración podría ser evapotranspirada luego de pocos días del evento de lluvia, y este proceso podría incluso acelerarse en parcelas con mayor temperatura y mayor cobertura vegetal debido a una mayor evapotranspiración (Austin et al. 2004). En este sentido, la evaluación del efecto de los pulsos de agua sobre el patrón de descomposición y la duración del efecto del pulso de lluvia sobre la humedad en la broza y en los primeros centímetros del suelo sería un punto importante a considerar en futuros estudios (Austin et al. 2004).

El patrón de descomposición delos materiales comunes presentó diferencias significativas entre las configuraciones vegetales analizadas, al igual que en otros trabajos (Quested et al. 2007; Zhang et al. 2008). Sin embargo, el patrón observado fue disímil entre los dos años de estudio para ambos materiales (sólo el arbustal abierto presentó mayor descomposición en ambos años), por lo que los mecanismos que se discuten a continuación podrían variar en importancia interanualmente. Por otro lado, los cambios en el ambiente local fueron tres veces mayores sobre la descomposición de A. communis que en J. ichu (coeficiente de variación de 21.6 y 6.8, respectivamente). Por lo tanto, las variaciones del ambiente local de descomposición, a causa de una mayor intensidad de uso de la tierra, serían más relevantes en comunidades vegetales dominadas por especies de descomposición rápida. Como se dijo antes, a nivel de parcela, la cobertura vegetal y la temperatura del aire se asociaron de forma negativa con la descomposición de los materiales comunes. Es posible que en las parcelas donde la cobertura vegetal, la temperatura del aire y del suelo son mayores, el perfil superficial del suelo permanezca húmedo por menos tiempo luego de un pulso de agua (Berg and Mc Claugherty 2014). Por este motivo, es probable que los microorganismos de los sitios con mayor cobertura vegetal estén expuestos a eventos de aridez más prologados (que no pudieron ser detectados por las mediciones de humedad instantánea realizadas en este estudio) y, por lo tanto, se vea afectada su actividad. Por otro 
lado, en las parcelas donde la cobertura vegetal y la cantidad de broza acumulada sobre el suelo es menor, el material a descomponer (broza o en nuestro caso materiales comunes) estaría expuesto a mayor radiación solar y a una mayor degradación como consecuencia de la fotodegradación (Gallo et al. 2009; Ndagurwa et al. 2015).

Si bien en el presente trabajo no se analizó el efecto de la fotodegradación de manera explícita, varios trabajos muestran que este proceso puede alcanzar valores entre $33 \mathrm{y}$ $60 \%$ de pérdida de peso en zonas semiáridas y destacan su importancia en el proceso de descomposición (Austin and Vivanco 2006; Baker and Allison 2015; Day et al. 2015). Estos autores proponen que la pérdida de carbono a través de la fotodegradación en estos ecosistemas implica que una fracción importante del C fijado en la biomasa de las plantas se pierde directamente a la atmósfera sin pasar antes a formar parte del C del suelo (Austin and Vivanco 2006). Si bien las pérdidas de C en los suelos de los arbustales abiertos se deben principalmente a una reducción de la biomasa vegetal (Conti et al. 2014) y a la mayor erosión eólica e hídrica de los suelos debido a una menor protección física (Martínez-Mena et al. 2002), la vía más corta en el ciclo de C producida por la fotodegradación puede ser una razón adicional por la cual el ingreso de C a los suelos sea menor (a pesar de una mayor descomposición potencial de los materiales comunes en los arbustales abiertos). Otros procesos como la fragmentación física de la broza causada por el viento, la lluvia y las heladas también podrían afectar el proceso de descomposición en ecosistemas áridos y semiáridos (Steinberger et al. 1990; Vanderbilt et al. 2008). Por otro lado, trabajos realizados en otros ecosistemas con distintos usos de la tierra han encontrado una disminución de la comunidad de microorganismos en los suelos de comunidades vegetales con usos de la tierra más intensos (Lagerlöf et al. 2014; Hortal et al. 2015; Smith et al. 2015). No se puede descartar la posibilidad que, dados los cambios en el ambiente local encontrados en el presente trabajo, se produzca un cambio en la comunidad de microorganismos que pueda relacionarse con la diferencia en la descomposición de los materiales comunes en las configuraciones vegetales.

Finalmente, pese a que el contenido de C y $\mathrm{N}$ del suelo difirió entre las configuraciones vegetales y se asoció a los cambios en la cobertura de la vegetación y en la cantidad de broza, los patrones de descomposición de los materiales comunes no se asociaron a la disponibilidad de dichos nutrientes. Por otro lado, según varios autores (Bääth et al. 1981; Blagodatskaya and Anderson 1998), el $\mathrm{pH}$ es una de las principales variables que controla la composición de la comunidad de microorganismos del suelo. Estos autores proponen que en suelos más ácidos predominan los hongos, mientras que en suelos más básicos predominan las bacterias. Si bien se detectó una relación del $\mathrm{pH}$ del suelo y la descomposición de los materiales comunes, en todas las parcelas analizadas los valores de $\mathrm{pH}$ encontrados fueron superiores a 5.4, por lo que pueden ser considerados suelos en general básicos dominados por bacterias. Consideramos que la mayor descomposición de los materiales comunes en las parcelas con valores de $\mathrm{pH}$ más bajos, no se deben a cambios de $\mathrm{pH}$ y sus efectos asociados sobre la comunidad de microorganismos del suelo, sino a los factores relacionados con los cambios de la cobertura vegetal. La falta de asociación entre la descomposición y las variables características de los suelos relacionadas a la disponibilidad de nutrientes, estaría indicando que no ejercen un efecto directo sobre los patrones de descomposición en las configuraciones estudiadas.

En general, en el Chaco Semiárido, el cambio en el uso de la tierra tiene un impacto muy evidente sobre la cobertura de la vegetación, pero este impacto no se traduce en cambios consistentes en el ambiente local de descomposición (medido a través de la descomposición de materiales comunes). En estudios futuros sería interesante evaluar la contribución relativa de los procesos de degradación abiótica (fotodegradación y fragmentación física) sobre la descomposición y el ciclado de nutrientes en general como así también la relación entre la liberación de $\mathrm{C}$ a la atmósfera (a partir de la materia muerta) vs. su almacenamiento en el suelo de estos sistemas semiáridos.

Agradecimientos. Agradecemos a nuestro equipo de investigación que amablemente ayudó en la preparación y el procesamiento de las muestras. También queremos agradecer a los revisores por sus importantes aportes en el presente manuscrito. Esta investigación se realizó con el apoyo de los siguientes subsidios: FONCyT (PICT 2004-16 / 25712, PICT 2008-365 y PICT 554) y CONICET PIP 2008-2013 / 11220080101532CO. Al mismo tiempo forma parte del proyecto SPG-CRA 2015 (que son apoyados por subsidios de NSF (EE.UU.) GEO-0452325 y GEO-1138881). 


\section{REFERENCIAS}

Abril, A., and E. H. Bucher. 1999. The effects of overgrazing on soil microbial community and fertility in the Chaco dry savannas of Argentina. Appl Soil Ecol 12(2):159-167.

Adams, J. 2007. Vegetation - Climate Interaction. How Vegetation Makes the Global Environment. Springer, Praxis Publishing Ltd, Chichester, UK.

Anderson-Teixeira, K. J., P. K. Snyder, T. E. Twine, S. V. Cuadra, M. H. Costa, and E. H. DeLucia. 2012. Climate-regulation services of natural and agricultural eco regions of the Americas. Nat Climate Change 2(3):177-181.

Arx, G., E. Graf Pannatier, A. Thimonier, and M. Rebetez. 2013. Microclimate in forests with varying leaf area index and soil moisture: potential implications for seedling establishment in a changing climate. J Ecol 101(5):1201-1213.

Austin, A. T., L. Yahdjian, J. M. Stark, J. Belnap, A. Porporato, et al. 2004. Water pulses and biogeochemical cycles in arid and semiarid ecosystems. Oecologia 141(2):221-235.

Austin, A. T., and L. Vivanco. 2006. Plant litter decomposition in a semi-arid ecosystem controlled by photodegradation. Nature 442(7102):555-558.

Bääth, E., B. Lundgren, and B. Söderström. 1981. Effects of nitrogen fertilization on the activity and biomass of fungi and bacteria in a podzolic soil. Zentralbl Bakteriol Mikrobiol Hyg I Abt Orig C 2:90-98.

Baker, N. R., and S. D. Allison. 2015. Ultraviolet photodegradation facilitates microbial litter decomposition in a Mediterranean climate. Ecology 96(7):1994-2003.

Berg, B., and C. McClaugherty. 2014. Decomposer organisms. Pages 35-52 en Plant Litter. Springer Berlin Heidelberg.

Blagodatskaya, E. V., and T. H. Anderson. 1998. Interactive effects of $\mathrm{pH}$ and substrate quality on the fungal to bacterial ratio and $\mathrm{CO}^{2}$ of microbial communities in forest soils. Soil Biol Biochem 30(10):1269-1274.

Bremner, J. M. 1996. Nitrogen - Total. Part 3, Chapter 37 en D. L. Sparks (ed.). Methods of Soil Analysis. ASA, SSSA, CSSA, Madison WI.

Cabido, M., S. Díaz, and A. Acosta. 1992. La vegetación del Chaco Árido en el oeste de la provincia de Córdoba, Argentina. Doc Phytosociol 14:447-456.

Cabido, M., and J. M. Pacha. 2002. Vegetación y flora de la Reserva Natural Chancaní. Agencia Córdoba Ambiente. Sección C. Publicaciones técnicas.

Conti, G., N. Pérez-Harguindeguy, F. Quètier, L. D. Gorné, P. Jaureguiberry, et al. 2014. Large changes in carbon storage under different land-use regimes in subtropical seasonally dry forests of southern South America. Agric Ecosyst Environ 197:68-76.

Cora, A., and O. A. Bachmeier. 2006. Número mínimo de muestras necesario para un muestreo edáfico en el Chaco Árido de Córdoba (Argentina). Multequina 15(2):97-102.

Cornelissen, J. H. C. 1996. An experimental comparison of leaf decomposition rates in a wide range of temperate plant species and types. J Ecol 84:573-582.

Coûteaux, M. M., P. Bottner, and B. Berg. 1995. Litter decomposition, climate and litter quality. Trends Ecol Evol 10: 63-66.

Crockatt, M. E., and D. P. Bebber. 2015. Edge effects on moisture reduce wood decomposition rate in a temperate forest. Glob Change Biol 21(2):698-707.

Cuchietti, A., E. Marcotti, D. E. Gurvich, A. M. Cingolani, and N. Pérez-Harguindeguy. 2014. Leaf litter mixtures and neighbour effects: low-nitrogen and high-lignin species increase decomposition rate of high-nitrogen and low-lignin neighbours. App Soil Ecol 82:44-51.

Cuchietti, A. 2016. Efectos del uso de la tierra y la biodiversidad funcional sobre el ciclado de la materia orgánica en el centro-oeste de Argentina. Doctor en Ciencias Biológicas. Universidad Nacional de Córdoba. Córdoba. Argentina. Pp. 24-28.

Day, P. R. 1986. Particle fractionation and particle-size analysis. Part I en D. L. Sparks (ed.). Methods of Soil Analysis. ASA, SSSA, CSSA, Madison WI.

Day, T. A., R. Guénon, and C. T. Ruhland. 2015. Photodegradation of plant litter in the Sonoran Desert varies by litter type and age. Soil Biol Biochem 89:109-122.

Deng, L., W. Yan, Y. Zhang, and Z. Shangguan. 2016. Severe depletion of soil moisture following land-use changes for ecological restoration: Evidence from northern China. Forest Ecol Manage 366:1-10.

Di Rienzo, J. A., R. E. Macchiavelli, and F. Casanoves. 2011. Modelos Mixtos en InfoStat. Primera edición. Pp. 197.

Di Rienzo, J. A., F. Casanoves, M. G. Balzarini, L. González, M. Tablada, et al. 2015. Grupo InfoStat, FCA, Universidad Nacional de Córdoba, Argentina. URL: www.infostat.com.ar.

Eviner, V. T., and F. S. Chapin III. 2003. Functional matrix: a conceptual framework for predicting multiple plant effects on ecosystem processes. Annu Rev Ecol Evol Syst 34:455-485.

Flerchinger, G. N., M. L. Reba, T. E. Link, and D. Marks. 2015. Modeling temperature and humidity profiles within forest canopies. Agr Forest Meteorol 213:251-262.

Furey, C., P. A. Tecco, N. Pérez-Harguindeguy, M. A. Giorgis, and M. Grossi. 2014. The importance of native and exotic plant identity and dominance on decomposition patterns in mountain woodlands of central Argentina. Acta Oecol 54:13-20.

Gallo, M. E., A. Porras-Alfaro, K. J. Odenbach, and R. L. Sinsabaugh. 2009. Photo acceleration of plant litter decomposition 
in an arid environment. Soil Biol Bioch 41(7):1433-1441.

Gorgas, J., and J. Tassile. 2003. Recursos naturales de la provincia de Córdoba. Los suelos. Agencia Córdoba Ambiente SE - INTA, Manfredi, Córdoba.

Harmon, M. E., O. N. Krankina, and J. Sexton. 2000. Decomposition vectors: a new approach to estimating woody detritus decomposition dynamics. Can J For Res 30(1):76-84.

Hättenschwiler, S., A. V. Tiunov, and S. Scheu. 2005. Biodiversity and litter decomposition in terrestrial ecosystems. Annu Rev Ecol Evol Syst 36:191-218.

Hobbie, S. E. 1992. Effects of plant species on nutrient cycling. Trends Ecol Evol 7(10):336-339.

Hortal, S., F. Bastida, J. L. Moreno, C. Armas, C. García, and F. I. Pugnaire. 2015. Benefactor and allelopathic shrub species have different effects on the soil microbial community along an environmental severity gradient. Soil Biol Biochem 88:48-57.

Houspanossian, J., M. Nosetto, and E. G. Jobbágy. 2013. Radiation budget changes with dry forest clearing in temperate Argentina. Glob Change Biol 19(4):1211-1222.

Hoyos, L. E., A. M. Cingolani, M. R. Zak, M. V. Vaieretti, D. E. Gorla, et al. 2013. Deforestation and precipitation patterns in the arid Chaco forests of central Argentina. App Veg Sci 16:260-271.

Jin, T. T., B. J. Fu, G. H. Liu, and Z. Wang. 2011. Hydrologic feasibility of artificial forestation in the semi-arid Loess Plateau of China. Hydrol Earth Syst Sci 15:2519-2530.

Karlin, M. S., U. O. Karlin, R. O. Coirini, G. J. Reati, and R. M. Zapata. 2013. El Chaco Árido. Universidad Nacional de Córdoba.

Kuo, S. 1996. Phosphorus. Part 3 en D. L. Sparks (ed.). Methods of Soil Analysis. ASA, SSSA, CSSA, Madison WI.

Lagerlöf, J., L. Adolfsson, G. Börjesson, K. Ehlers, G. P. Vinyoles, and I. Sundh. 2014. Land-use intensification and agroforestry in the Kenyan highland: impacts on soil microbial community composition and functional capacity. App Soil Ecol 82:93-99.

Lee, X., M. L. Goulden, D. Y. Hollinger, A. Barr, T. A. Black, et al. 2011. Observed increase in local cooling effect of deforestation at higher latitudes. Nature 479(7373):384-387.

Longobardi, A. 2008. Observing soil moisture temporal variability under fluctuating climatic conditions. Hydrol. Earth Syst Sc Disc 5(2):935-969.

Martínez-Mena, M., J. Álvarez Rogel, V. Castillo, and J. Albaladejo. 2002. Organic carbon and nitrogen losses influenced by vegetation removal in a semiarid mediterranean soil. Biogeochemistry 61:309-321.

Ndagurwa, H. G., J. S. Dube, and D. Mlambo. 2015. Decomposition and nutrient release patterns of mistletoe litters in a semiarid savanna, southwest Zimbabwe. Austral Ecol 40(2):178-185.

Nelson, D. W., and L. E. Sommers. 1996. Total Carbon, Organic Carbon, and Organic Matter. Part 3, Chemical Methods. En D. L Sparks (ed.). Methods of Soil Analysis. ASA, SSSA, CSSA, Madison WI.

O'Lear, H. A., T. R. Seastedt, J. M. Briggs, J. M. Blair, et al. 1996. Fire and topographic effects on decomposition rates and nitrogen dynamics of buried wood in tall grass prairie. Soil Biol Bioch 28:322-329.

Orwin, K. H., D. A. Wardle, G. Laurence, and L. G. Greenfield. 2006. Ecological consequences of carbon substrate identity and diversity in a laboratory study. Ecology 87:580-93.

Paudel, E., G. G. Dossa, J. Xu, and R. D. Harrison. 2015. Litterfall and nutrient return along a disturbance gradient in a tropical montane forest. For Ecol Manag 353:97-106.

Pérez-Harguindeguy, N., S. Díaz, J. H. Cornelissen, and M. Cabido. 1997. Comparación experimental de la tasa de descomposición foliar de especies vegetales del centro-oeste de Argentina. Ecol Austral 7:87-94.

Pérez-Harguindeguy, N., S. Díaz, E. Garnier, S. Lavorel, H. Poorter, et al. 2013. New handbook for standardised measurement of plant functional traits worldwide. Aust J Bot 61:167-234.

Prescott, C. E. 2010. Litter decomposition: what controls it and how can we alter it to sequester more carbon in forest soils? Biogeochemistry 101(1-3):133-149.

Quested, H., O. Eriksson, C. Fortunel, and E. Garnier. 2007. Plant traits relate to whole-community litter quality and decompositon following land use change. Func Ecol 21:1016-1026.

Rundel, P., and W. Jarrell. 1989. Water in the environment. Pp. 29-56 en R. W. Pearcy, J. Ehleringer, H. A. Mooney and P. Rundel. Plant Physiological Ecology. Chapman y Hall, New York, New York.

Schilling, J. S., A. Ayres, J. T. Kaffenberger, and J. S. Powers. 2015. Initial white rot type dominance of wood decomposition and its functional consequences in a regenerating tropical dry forest. Soil Biol Biochem 88:58-68.

Seidelmann, K. N., M. Scherer-Lorenzen, and P. A. Niklaus. 2016. Direct vs. Microclimate-Driven Effects of Tree Species Diversity on Litter Decomposition in Young Subtropical Forest Stands. PloS One 11(8):e0160569.

Smith, A. P., E. Marín Spiotta, and T. Balser. 2015. Successional and seasonal variations in soil and litter microbial community structure and function during tropical postagricultural forest regeneration: a multiyear study. Glob Chang Biol 21(9):3532-3547.

Sokal, R. R., and F. J. Rohlf. 1995. Biometry. W.H. Freeman and Company, New York.

Steinberger, Y., A. Shmida, and W. G. Whitford. 1990. Decomposition along a rainfall gradient in the Judean desert, Israel. Oecologia 82(3):322-324.

Thomas, G. W. 1996. Soil pH and soil acidity. Part 3. Chapter 16. En D. L. Sparks (ed.). Methods of Soil Analysis. ASA, SSSA, CSSA, Madison WI. 
Vaieretti, M. V., N. Pérez Harguindeguy, D. E. Gurvich, A. M. Cingolani, and M. Cabido. 2005. Decomposition dynamics and physico-chemical leaf quality of abundant species in a montane woodland in central Argentina. Plant Soil 278: 223-234.

Vaieretti, M. V., A. M. Cingolani, N. Pérez-Harguindeguy, D. E. Gurvich, and M. Cabido. 2010. Does decomposition of standard materials differ among grassland patches maintained by livestock? Austral Ecol 35(8):935-943.

Vanderbilt, K. L., C. S. White, O. Hopkins, and J. A. Craig. 2008. Aboveground decomposition in arid environments: results of a long-term study in central New Mexico. J Arid Environ 72(5):696-709.

Vivanco, L., and A. T. Austin. 2006. Intrinsic effects of species on leaf litter and root decomposition: a comparison of temperate grasses from North and South America. Oecologia 150(1):97-107.

Vivanco, L., and A. T. Austin. 2008. Tree species identity alters forest litter decomposition through long term plant and soil interactions in Patagonia, Argentina. J Ecol 96(4):727-736.

Yates, C. J., D. A. Norton, and R. J. Hobbs. 2000. Grazing effects on plant cover, soil and microclimate in fragmented woodlands in southwestern Australia: implications for restoration. Austral Ecol 25(1):36-47.

Zak, M. R., M. Cabido, D. Cáceres, and S. Díaz. 2008. What drives accelerated land cover change in central Argentina? Synergistic consequences of climatic, socioeconomic, and technological factors. Environ Manage 42(2):181-189.

Zhang, D., D. Hui, and G. Zhou. 2008. Rates of litter decomposition in terrestrial ecosystems: global patterns and controlling factors. J Plant Ecol 1(2):85-93.

Zuloaga, F. O., O. Morrone, and M. J. Belgrano. 2008. Catalogue of the vascular plants of the southern cone (Argentina, southern Brazil, Chile, Paraguay and Uruguay). Volume 2: Dicotyledoneae: Acanthaceae-Fabaceae (AbaremaSchizolobium) (Pp. xx+-985). Missouri Botanical Garden Press. 